„TECHNISCH GESEHEN WAR ES NOCH NIE SO EINFACH WIE HEUTE, WISSENSCHAFTLER UND SCHÜLER ZU VERNETZEN. EIN VIDEOTELEFONAT IM UNTERRICHT KANN WISSEN-

SCHAFT ERLEBBAR MACHEN. LASST UNS DIE VIRTUELLEN TÜREN VON LABOREN ÖFFNEN!“

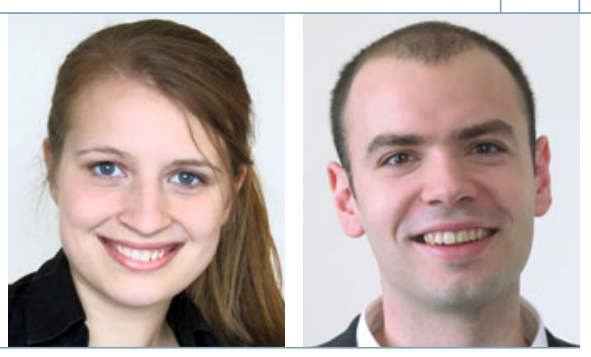

Kerstin Göpfrich und Karl Gödel

\title{
Ring-a-Scientist - der direkte Draht ins Labor
}

DOI: $10.1007 / \mathrm{s} 12268-018-0944-3$

(C) Springer-Verlag 2018

Wissenschaft gehört ins Klassenzimmer! Nur so lassen sich Schülerinnen und Schüler frühzeitig und kontinuierlich für technischnaturwissenschaftliche Fächer begeistern, nur so lässt sich der Fachkräftemangel abbauen. Nur so werden junge Menschen zu mündigen Bürgern, die die Chancen und Risiken von zukunftsweisenden Technologien im gesellschaftlichen Dialog verantwortungsvoll abwägen. Und nur so kann das Vertrauen der Öffentlichkeit in die Wissenschaft gestärkt werden - das erst kürzlich durch die Debatte um Raubverleger und wissenschaftliche Pseudojournale erneut erschüttert wurde. Aktuelle Themen stoßen im Unterricht häufig auf großes Interesse, doch sie einzubringen, kann nicht allein Aufgabe von Lehrerinnen und Lehrern sein. Auch Studierende und Forschende sind gefragt. Doch wer eine Schulklasse besucht, ist oftmals einen ganzen Tag unterwegs. Der Organisationsaufwand von Laborbesuchen ist kaum geringer - Zeit, die nicht nur im Alltag als Wissenschaftler, sondern auch in den Lehrplänen der Schulen schlichtweg fehlt. Mehr als ein Besuch pro Jahr ist kaum realistisch - obgleich eine kontinuierliche Interaktion zwischen Schule und Wissenschaft doch so wichtig und gewinnbringend sein könnte.

Technisch gesehen gibt es längst eine Lösung für das Dilemma: virtuelle Besuche per Webvideokonferenz. Eine Videokonferenz lässt sich ohne größeren Aufwand in eine Unterrichtsstunde integrieren und ist auch mit dem Alltag in der Forschung gut vereinbar. Wir Wissenschaftler kommunizieren ohnehin häufig über Kommunikationsdienste wie Skype und Co. Der Zeitaufwand beschränkt sich auf kaum eine Stunde, und kostengünstiger ist die virtuelle Kommunikation obendrein. Außerdem ergeben sich ganz neue Möglichkeiten: Es können Laborexperimente gezeigt werden, die sich nicht ins Klassenzimmer transportieren ließen wie ein DNA-Sequenzierer in der Biologie oder ein Elektronenstrahlmikroskop im Physikunterricht. Wir können Schulen im ländlichen Raum erreichen, weit über das lokale Umfeld hinaus. Und die Schülerinnen und Schüler scheinen sich in der virtuellen Interaktion ohnehin wohlzufühlen: Sie stellen Fragen, die sie in einem persönlichen Gespräch vielleicht gescheut hätten oder bitten um Anschlussgespräche. Dennoch sollten Besuche niemals wegfallen, sondern nur ergänzt werden.

Damit Videotelefonate ein fester Bestandteil im Unterricht werden, gilt es Lehrer und Wissenschaftler global zu vernetzen. Genau dieses Ziel verfolgt www.ring-a-scientist.org eine Webplattform, über die Lehrer unbürokratisch Termine für Videokonferenzen mit Wissenschaftlern vereinbaren können. Interessierte Forschende aller Fachdisziplinen vom Erstsemestler in Geschichte bis zur Biologie-Professorin, vielleicht auch der ein oder andere Leser - können auf der Plattform ein Profil anlegen. Direkt dort versenden Lehrerinnen und Lehrer dann ihre Terminanfrage mit dem Gesprächswunsch. Mögliche Gesprächsinhalte reichen von Studienberatung und Diskussionsrunden mit Expertinnen und Experten hin zu virtuellen Laborführungen, Experimenten und Einblicken in die aktuelle Forschung. Durch die Live-Konferenzen vom Labor ins Klassenzimmer wird Wissenschaft als Prozess erlebbar. Der aktive Austausch mit Forschenden soll Schülerinnen und Schüler bestärken, Wissen nicht nur passiv aufzunehmen, sondern in einem kollaborativen Prozess zu nutzen und zu mehren. Langfristig werden nicht nur Schulen profitieren, denn Web-Videokonferenzen lassen sich auch anderweitig einsetzen: Zum Beispiel können im Museum die Forschenden der Datenbank bald als „Live-Exponat“ zu sehen sein. Ring$a$-Scientist will so einen Beitrag leisten zur Chancengleichheit beim Zugang zu Wissen und Bildung.
Auch die Wissenschaftlerinnen und Wissenschaftler profitieren von dem Einsatz der Web-Videotelefonie: Schließlich ist Zeitmangel der am häufigsten genannte Grund, wieso die Kommunikation gescheut wird. Gleichzeitig wird ein schlüssiges Kommunikationskonzept auch in Anträgen um Drittmittel zu Recht immer gewichtiger. Die Frage nach der Verantwortung des Wissenschaftlers ist heute aktueller denn je - sich ihr zu entziehen, würde langfristig allen Seiten schaden. Lassen Sie uns deshalb die virtuellen Türen unserer Labore öffnen! Wir wünschen uns ein weltweites Netzwerk, damit es ganz selbstverständlich wird, Experten oder Laborexperimente regelmäßig in interaktive Unterrichtsstunden einzubeziehen. Beteiligen Sie sich!

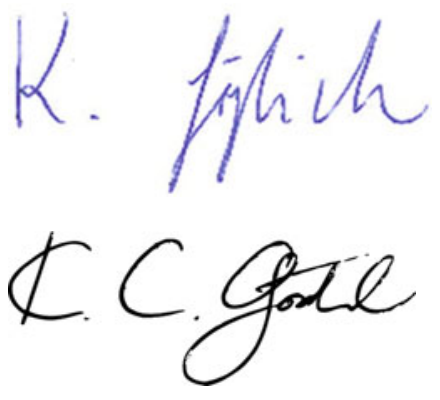

Kerstin Göpfrich, Karl Gödel, www.ring-a-scientist.org

Korrespondenzadresse:

Dr. Kerstin Göpfrich

Dr. Karl Gödel

Emilienstraße 16A

D-70563 Stuttgart

Tel.: 0176-43232580

mail@ring-a-scientist.org

www.ring-a-scientist.org 